Portland State University

PDXScholar

$1-21-2016$

\title{
Performance Metrics for Depth-Based Signal Separation using Deep Vertical Line Arrays
}

\author{
Gabriel Paul Kniffin \\ Portland State University \\ John Kenneth Boyle \\ Portland State University \\ Lisa Zurk \\ Portland State University, zurkl@pdx.edu \\ Martin Siderius \\ Portland State University, siderius@pdx.edu
}

Follow this and additional works at: https://pdxscholar.library.pdx.edu/ece_fac

Part of the Acoustics, Dynamics, and Controls Commons, and the Civil and Environmental Engineering Commons

\section{Let us know how access to this document benefits you.}

\section{Citation Details}

Kniffin, G. P., Boyle, J. K., Zurk, L. M., \& Siderius, M. (2016). Performance metrics for depth-based signal separation using deep vertical line arrays. The Journal of the Acoustical Society of America, 139(1), 418.

This Article is brought to you for free and open access. It has been accepted for inclusion in Electrical and Computer Engineering Faculty Publications and Presentations by an authorized administrator of PDXScholar. Please contact us if we can make this document more accessible: pdxscholar@pdx.edu. 


\title{
Performance metrics for depth-based signal separation using deep vertical line arrays
}

\author{
Gabriel P. Kniffin, a) John K. Boyle, Lisa M. Zurk, and Martin Siderius \\ Department of Electrical and Computer Engineering, Northwest Electromagnetics and Acoustics Research \\ Laboratory (NEAR-Lab), Portland State University, Portland, Oregon 97207, USA
}

(Received 21 August 2015; revised 10 December 2015; accepted 28 December 2015; published online 21 January 2016)

\begin{abstract}
A recent publication by McCargar and Zurk [(2013). J. Acoust. Soc. Am. 133(4), EL320-EL325] introduced a modified Fourier transform-based method for passive source depth estimation using vertical line arrays deployed below the critical depth in the deep ocean. This method utilizes the depth-dependent modulation caused by the interference between the direct and surface-reflected acoustic arrivals, the observation of which is enhanced by propagation through the reliable acoustic path. However, neither the performance of this method nor its limits of applicability have yet been thoroughly investigated. This paper addresses both of these issues; the first by identifying and analyzing the factors that influence the resolution and ambiguity in the transform-based depth estimate; the second by introducing another, much simpler depth estimation method, which is used to determine the target trajectories required for observation of the interference pattern and the array requirements for accurate depth estimation. (C) 2016 Acoustical Society of America.
\end{abstract}

[http://dx.doi.org/10.1121/1.4939740]

$[\mathrm{ZHM}]$

Pages: 418-425

\section{INTRODUCTION}

At low frequencies, the primary impediment to the detection of quiet submerged sources is the ambient noise due to surface ship traffic. ${ }^{1}$ However, recent work has shown that vertical line arrays (VLAs) deployed below the critical depth - the depth below the channel axis at which the sound speed is equal to the maximum sound speed near the surface-benefit from propagation effects that significantly reduce distant shipping noise. ${ }^{2,3}$ In addition, sound from sources at moderate ranges undergo propagation via the reliable acoustic path (RAP), ${ }^{4}$ which may result in a $10-20 \mathrm{~dB}$ reduction in transmission loss (TL) compared to a shallow receiver at the same range. ${ }^{5}$ However, these favorable propagation conditions also apply to nearby surface interferers, and while a deep VLA with sufficient resolution can separate such moderate-range sources from distant shipping noise in vertical angle, the lack of horizontal aperture prohibits azimuthal rejection. Furthermore, matched-field methods that have been proposed for depth discrimination with VLAs to address this challenge are hindered by environmental uncertainty. ${ }^{6,7}$

One promising alternative takes advantage of the Lloyd's mirror interference pattern that arises from the coherent summation of the direct and surface-reflected acoustic paths. ${ }^{5,8}$ Previous work by the authors ${ }^{5,8,9}$ has shown that even in complex ocean environments, the dominant structure of this interference pattern is captured with a fairly simple image theory expression that can be derived from either modal or ray propagation expressions. Subsequent work $^{10}$ has used this concept of an interference pattern and related it

a)Electronic mail: kniffing@pdx.edu to the wavenumber spectrum, essentially deriving the same basic structure. The interference introduces depth-harmonic modulation in the signals from submerged targets, providing a means of depth-based signal separation using a modified Fourier transform. ${ }^{5,8,9}$ A more recent method based on an extended Kalman filter also utilizes the coherent summation of direct and surface-reflected acoustic arrivals recorded on a single hydrophone. ${ }^{11}$ However, that method uses multiple observations coupled with a motion hypothesis to compensate for the lack of array aperture, as well as processing gain obtained from a known pseudorandom source signal, which is not applicable to passive detection.

In this paper, the factors influencing the performance and limits of applicability of the modified Fourier transformbased depth estimation method are investigated. Section II introduces the physical origin and geometrical characteristics of the depth-harmonic interference structure. Section III then provides an overview of the transform-based depth estimation method developed in previous work, ${ }^{5,8,9}$ and a new characterization of its performance in terms of the resolution and ambiguity in the depth estimate. Finally, Sec. IV introduces a new simplified depth estimation method based on the observed spacing of the depth-harmonic interference nulls, which is used to determine the target trajectories necessary for observation of the modulation, as well as the VLA requirements that provide resolution of the null spacing sufficient for depth estimation.

\section{DEPTH-HARMONIC STRUCTURE}

Figure 1 illustrates the problem geometry wherein a VLA near the ocean bottom, centered at depth $\bar{z}$, is insonified by a submerged source at constant depth $z_{s}$ emitting a tone at frequency $\omega=2 \pi f$. For a target traveling in a straight line 
course past the array at a constant velocity $v_{s}$, such as in a shipping channel, the time-dependent surface range to the VLA is given by

$$
r_{s}(t)=\sqrt{r_{\mathrm{CPA}}^{2}+v_{s}^{2}\left(t-t_{\mathrm{CPA}}\right)^{2}},
$$

where $r_{\mathrm{CPA}}$ and $t_{\mathrm{CPA}}$ are the surface range and time at the source's closest point of approach (CPA). The slant ranges from the direct and surface-reflected paths of the submerged source and its image, shown in Fig. 1, are given by

$$
R_{s}^{ \pm}(t)=\sqrt{r_{s}^{2}(t)+\left(\bar{z} \mp z_{s}\right)^{2}}
$$

From image theory, the received pressure on the VLA at time $t$ is given by

$$
p(z, \omega, t)=S(\omega)\left[\frac{e^{i k R_{s}^{+}(t)}}{R_{s}^{+}(t)}+\Gamma \frac{e^{i k R_{s}^{-}(t)}}{R_{s}^{-}(t)}\right],
$$

where time dependence $e^{-i \omega t}$ has been suppressed for simplicity, the wavenumber in the environment with sound speed $c$ is given by $k=\omega / c$, the complex spectral amplitude is given by $S(\omega)$, and $\Gamma$ is the surface reflection coefficient. While an actual deep ocean environment will likely introduce bottom reflections and a reduction in the vertical arrival angles from signals from distant sources due to the variable sound speed profile, previous work $^{5,8,9}$ has shown results from normal mode $\left(\mathrm{KRAKEN}^{12}\right)$ simulations that include these effects. Within the RAP zone, sound propagates at steep angles relative to the horizontal, resulting in little sound speed profile-induced change in the path length difference between direct and surface-reflected acoustic arrivals. Hence, the results of image theory provide an accurate-but simple-framework to describe the inference pattern mathematically. Application of more advanced propagation theory ${ }^{5,8-10}$ adds complexity, but reduces to the same essential expression.

Due to high acoustic impedance contrast, the air-sea interface can be approximated as a pressure release boundary.

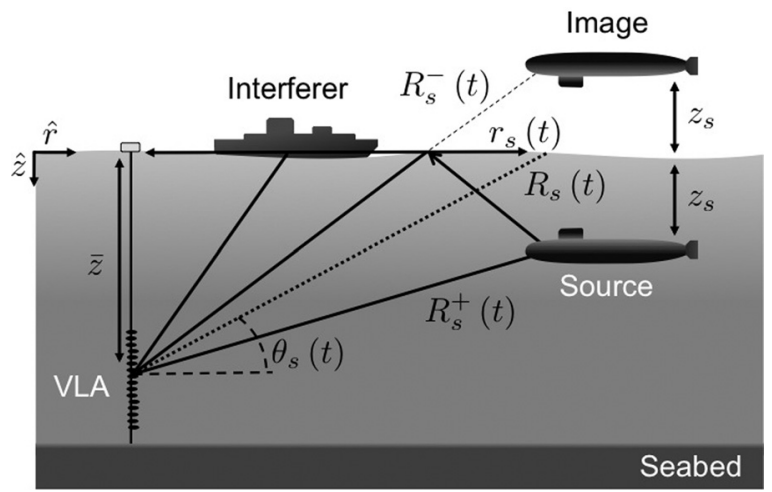

FIG. 1. The general geometry for received passive signals on a deep VLA (not shown to scale). Sound from a submerged source at surface range $r_{s}(t)$ and depth $z_{s}$ is observed on the the VLA whose center lies at depth $\bar{z}$. From image theory, the direct and surface-reflected arrivals correspond to slant ranges $R_{s}^{+}(t)$ and $R_{s}^{-}(t)$, respectively, with slant range $R_{s}(t)$ and vertical angle $\theta_{s}(t)$ corresponding to the midpoint between the source and its image.
In addition, the steep reflection angles in the RAP zone result in negligible rough surface scattering at low frequencies. Under these conditions, the surface reflection coefficient $\Gamma \approx$ -1 in Eq. (3). For a sufficiently deep VLA (i.e., below the critical depth), $\bar{z} \gg z_{s}$, and Eq. (2) can be approximated as

$$
R_{s}^{ \pm}(t) \approx R_{s}(t)=\sqrt{r_{s}^{2}(t)+\bar{z}^{2}}
$$

which allows Eq. (3) to be approximated as

$$
p(z, \omega, t) \approx-2 i S(\omega) \frac{e^{i k R_{s}(t)}}{R_{S}(t)} \sin \left(k z_{s} \sin \theta_{s}(t)\right),
$$

where

$$
\sin \theta_{s}(t)=\frac{\bar{z}}{R_{s}(t)}=\frac{\bar{z}}{\sqrt{\bar{z}^{2}+r_{\mathrm{CPA}}^{2}+v_{s}^{2}\left(t-t_{\mathrm{CPA}}\right)^{2}}}
$$

Assuming sufficient vertical aperture, $\sin \theta_{s}(t)$ can be determined by evaluating the time-varying output of a planewave beamformer, referred to as a vertical time record (VTR). This is analogous to a bearing time record (BTR) commonly used to show the time-varying output of a horizontal line array beamformer. Figure 2 shows two example simulated VTRs, generated with KRAKEN, for a $150 \mathrm{~Hz}$ source at different depths: $z_{s}=1 \mathrm{~m}$ in Fig. 2(a) and $z_{s}=50 \mathrm{~m}$ in Fig. 2(b). Both cases assume a noiseless environment with a refracting Munk ${ }^{13}$ sound speed profile and a single target traveling on a straight line course with parameters listed in Table I. Figure 2 also shows solid (a) and dashed (b) lines corresponding to the approximate target track, sin $\theta_{\hat{s}}(t)$, which in this case is given by the true target position from Eq. (6), shifted downward by -0.025 in $\sin \theta$ to correct for the refraction introduced by the Munk sound speed profile

$$
\sin \theta_{\hat{s}}(t) \equiv \sin \theta_{s}(t)-0.025 \text {. }
$$

In an actual passive sonar system, traces along a target track may be selected manually by a human sonar operator or automatically by a detection/tracking algorithm. Once a target trace $\sin \theta_{\hat{s}}(t)$ has been determined, the beamformer can be steered to it, resulting in a trace signal given approximately by b $^{5,9}$

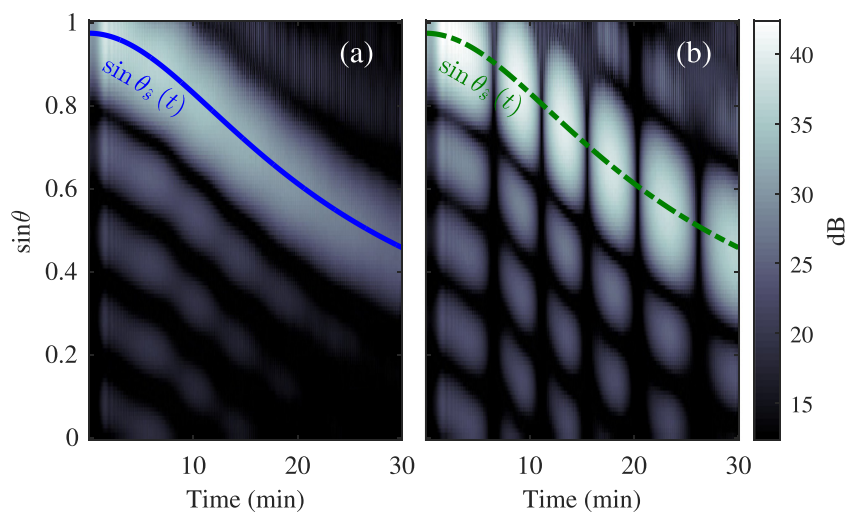

FIG. 2. (Color online) Example of depth-dependent modulation in a VTR at $150 \mathrm{~Hz}$ for source depth of (a) $1 \mathrm{~m}$ and (b) $50 \mathrm{~m}$. Solid and dashed lines on (a) and (b), respectively, show the target track $\sin \theta_{\hat{s}}(t)$. 
TABLE I. Normal mode $\left(\mathrm{KRAKEN}^{12}\right)$ simulation parameters used in generating VTR data shown in Fig. 2.

\begin{tabular}{lcc}
\hline \hline Description & Symbol & Value \\
\hline Source frequency & $f$ & $150 \mathrm{~Hz}$ \\
Source level & $\mathrm{SL}$ & $110 \mathrm{~dB} \mathrm{re} 1 \mu \mathrm{Pa}$ \\
Source depth & $z_{s}$ & (a) $1 \mathrm{~m},(\mathrm{~b}) 50 \mathrm{~m}$ \\
Surface range at source CPA & $r_{\mathrm{CPA}}$ & $20 \mathrm{~m}$ \\
Time at source CPA & $t_{\mathrm{CPA}}$ & $0 \mathrm{~s}$ \\
Source velocity & $v_{s}$ & $5 \mathrm{~m} / \mathrm{s}$ \\
Array center depth & $\bar{z}$ & $4972.5 \mathrm{~m}$ \\
Time snapshot length & $\Delta t$ & $3.6 \mathrm{sec}$ \\
Array elements & $N$ & 10 \\
Array spacing & $d$ & $5 \mathrm{~m}$ \\
Water column depth & - & $5000 \mathrm{~m}$ \\
\hline
\end{tabular}

$$
\begin{aligned}
& B\left(\omega, \sin \theta_{\hat{s}}(t)\right) \\
& \quad=2 \frac{|S(\omega)|^{2}}{\bar{z}^{2}} \sin ^{2} \theta_{\hat{s}}(t)\left(1-\cos \left(2 k z_{s} \sin \theta_{\hat{s}}(t)\right)\right) .
\end{aligned}
$$

Figure 3 shows two such trace signals corresponding to the solid and dashed traces shown in Figs. 2(a) and 2(b), respectively. The trace signal from the submerged source $\left(z_{s}=50 \mathrm{~m}\right)$ is easily distinguishable from the signal from the source at the surface $\left(z_{s}=1 \mathrm{~m}\right)$ due to the depth-harmonic modulation. This modulation is encapsulated in the cosine term in Eq. (8), which shows the modulation is periodic in $\sin \theta$ with period

$$
\sin \theta_{\text {period }}=\frac{c}{2 f z_{s}} .
$$

While the presence of the interference structure in the trace signal automatically indicates a submerged target, the source depth may also be estimated using the recently developed transform-based method, ${ }^{5,8}$ which is reviewed and analyzed in Sec. III. A simplified method based on directly observing the null spacing [Eq. (9)] is then introduced in Sec. IV. The null spacing-based method also provides a convenient means of quantitatively determining target

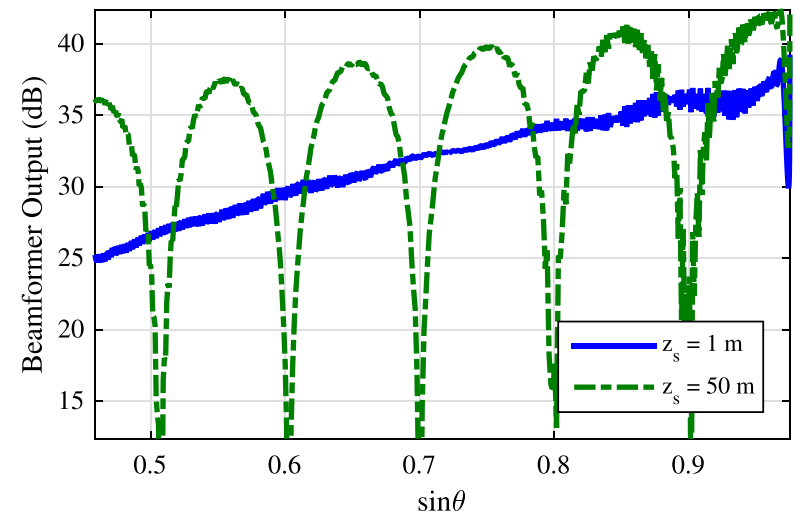

FIG. 3. (Color online) Trace signals corresponding to the solid line in Fig. 2(a) from the surface source VTR $\left(z_{s}=1 \mathrm{~m}\right)$ and dashed line in Fig. 2(b) from the submerged source VTR $\left(z_{s}=50 \mathrm{~m}\right)$. Depth-harmonic modulation provides a means of distinguishing submerged sources from sources at the surface. trajectory requirements for observation of the interference pattern. It can also be used to determine array requirements for sufficient resolution to accurately determine null locations for depth estimation. Analyses of both requirements are included in Sec. IV.

\section{TRANSFORM-BASED DEPTH ESTIMATION PERFORMANCE}

A transform-based method for estimating target depth was previously developed and demonstrated by the authors. ${ }^{5,8,9}$ This section reviews the transform-based method and describes and quantifies the factors that influence its performance in terms of the resolution and ambiguity in the depth estimate.

The transform itself is essentially a spatial Fourier synthesis in $z$,

$$
M(z)=\mathcal{F}\left\{m\left(k_{z}\right)\right\}=\int_{-\infty}^{\infty} m\left(k_{z}\right) e^{i k_{z} z} d k_{z},
$$

where

$$
k_{z}=k \sin \theta
$$

is the spatial frequency, corresponding to the $z$ component of the wave vector of a plane wave. ${ }^{14}$ Since $k_{z}$ encapsulates both the temporal frequency $(\omega)$ and angular $(\sin \theta)$ dependence, Eq. (8) can be reparameterized as

$$
B\left(k_{z \hat{s}}(t)\right)=2 \frac{|S(\omega)|^{2}}{k^{2} \bar{z}^{2}} k_{z \hat{s}}^{2}(t)\left(1-\cos \left(2 z_{s} k_{z \hat{s}}(t)\right)\right),
$$

where

$$
k_{z \hat{s}}(t) \equiv k \sin \theta_{\hat{s}}(t)
$$

and $\sin \theta_{\hat{s}}(t)$ is the approximate target track. Until this point, the formulations presented have been in continuous time. However, in a real passive sonar system, the signals will be sampled in time, which can be represented mathematically by multiplication by a Dirac comb function,

$$
\amalg(t)=\sum_{m} \delta(t-m \Delta t),
$$

where $m$ is the sample index and $\Delta t$ is the sampling interval. Substituting time $t_{m} \equiv m \Delta t$ into Eq. (6) gives rise to the sampling function in the $k_{z}$ domain,

$$
\amalg\left(k_{z \hat{s}}(t)\right)=\sum_{m} \delta\left(k_{z}-k_{z \hat{s}}\left(t_{m}\right)\right) .
$$

In addition, the trace signal is only observed over a finite interval in the $k_{z}$ domain, or equivalently, a finite interval in $\sin \theta$ as shown in Fig. 2 (the upper bound being $|\sin \theta| \leq 1$ ).

This finite extent can be represented by a rectangular window function

$$
\Pi\left(k_{z}\right)= \begin{cases}1 & \text { for } \min \left(k_{z}\right) \leq k_{z} \leq \max \left(k_{z}\right), \\ 0 & \text { otherwise }\end{cases}
$$


The net result is the trace signal in Eq. (12), sampled by Eq. (15), and windowed by Eq. (16),

$$
\begin{aligned}
& \Pi\left(k_{z \hat{s}}(t)\right) \amalg\left(k_{z \hat{s}}(t)\right) B\left(k_{z \hat{s}}(t)\right) \\
& \quad=A_{M}\left[k_{z \hat{s}}^{2}(t) \Pi\left(k_{z \hat{s}}(t)\right)\right]\left[\amalg\left(k_{z \hat{s}}(t)\right)\right]\left[1-\cos \left(2 z_{s} k_{z \hat{s}}(t)\right)\right],
\end{aligned}
$$

where $A_{M}=2|S(\omega)|^{2} / k^{2} \bar{z}^{2}$ is the modulation amplitude of Eq. (12). Taking the transform [given by Eq. (10)] of Eq. (17) and applying the convolution product theorem yields the threefold convolution,

$$
\begin{aligned}
M(z, \omega)= & \mathcal{F}\left\{\Pi\left(k_{z \hat{s}}(t)\right) \amalg\left(k_{z \hat{s}}(t)\right) B\left(k_{z \hat{s}}(t)\right)\right\} \\
= & A_{M} \mathcal{F}\left\{k_{z \hat{s}}^{2}(t) \Pi\left(k_{z \hat{s}}(t)\right)\right\} * \mathcal{F}\left\{\amalg\left(k_{z \hat{s}}(t)\right)\right\} \\
& *\left[\delta(z)-\frac{1}{2} \delta\left(z \pm 2 z_{s}\right)\right] .
\end{aligned}
$$

The first convolution term,

$$
\mathcal{F}\left\{k_{z \hat{s}}^{2}(t) \Pi\left(k_{z \hat{s}}(t)\right)\right\},
$$

therefore represents the spherical spreading loss $k_{z \hat{s}}(t)$ along with the windowing in Eq. (16) due to the limited track extent. Similarly, the second term,

$$
\mathcal{F}\left\{\amalg\left(k_{z \hat{s}}(t)\right)\right\},
$$

represents the effect of sampling by Eq. (15), while the third term,

$$
\left[\delta(z)-\frac{1}{2} \delta\left(z \pm 2 z_{s}\right)\right],
$$

is a sum of three delta functions corresponding to depths $z=0$ and $\pm 2 z_{s}$, which is utilized for depth estimation. $5,8,9$ For example, Fig. 4 shows the output of the transform applied to the trace signals in Fig. 3. In this case, the transform output shows two peaks corresponding to the delta functions at $z=0$ and $z=+z_{s}$ (the peak at $z=-z_{s}$ is outside the limits of the horizontal axis). For the case of $z_{s}=1 \mathrm{~m}$, the peak overlaps significantly with the peak at $z=0 \mathrm{~m}$, making

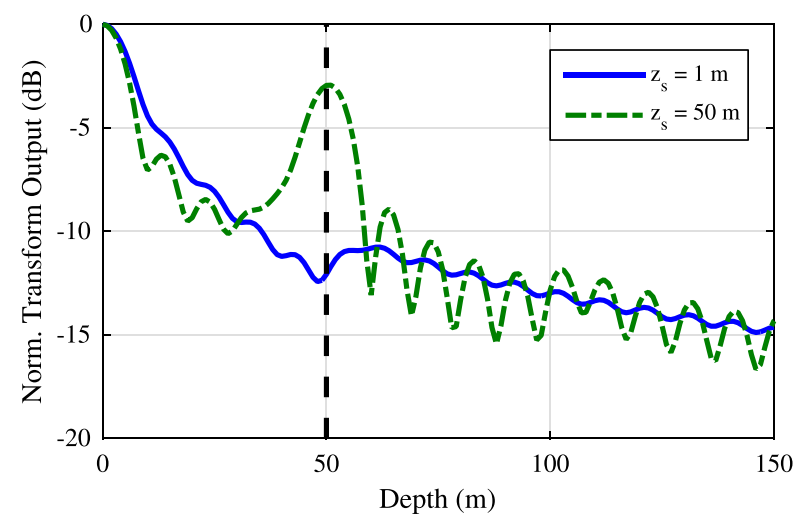

FIG. 4. (Color online) Normalized output of transform in Eq. (10) applied to trace signals in Fig. 3. The peak in the transform output corresponds to the $50 \mathrm{~m}$ depth of the submerged source, making it easily distinguishable from the source at $1 \mathrm{~m}$ depth. it unidentifiable without further high-pass filtering. ${ }^{5,8,9,15}$ In contrast, the peak corresponding to $z_{s}=50 \mathrm{~m}$ is easily distinguishable from the peak at $z=0 \mathrm{~m}$, clearly indicating the depth of the target. The amplitude of the $z_{s}=50 \mathrm{~m}$ is also $3 \mathrm{~dB}$ lower in amplitude than the $z=0 \mathrm{~m}$ peak due to the factor of $-1 / 2$ applied to the $\pm z_{s}$ delta function in the third term of Eq. (18).

As Secs. III A and III B will discuss in greater detail, the first two convolution terms, Eqs. (19) and (20), introduce artifacts which set limits on the resolution and introduce ambiguity in the transform-based depth estimate. However, it will be shown that these limits do not affect depth determination in most practical cases.

\section{A. Track extent and depth resolution}

The limited trace signal extent encapsulated in the window function [Eq. (16)] gives rise to the first convolution term [Eq. (19)], which acts to widen or "blur" the delta functions in equation (21). Parameterizing the window function in terms of the total target track extent

$$
K_{z}=\max \left(k_{z}\right)-\min \left(k_{z}\right)
$$

and center

$$
\bar{k}_{z}=\frac{\max \left(k_{z}\right)+\min \left(k_{z}\right)}{2},
$$

in the $k_{z}$ domain allows this "blurring function" to be expressed as

$$
\begin{aligned}
\mathcal{F}\left\{k_{z}^{2} \Pi\left(k_{z}\right)\right\} & =\int_{-\infty}^{\infty} k_{z}^{2} \Pi\left(\frac{k_{z}-\bar{k}_{z}}{K_{z}}\right) e^{i k_{z} z} d k_{z} \\
& =-K_{z} \frac{\partial^{2}}{\partial z^{2}} e^{i \bar{k}_{z} z} \operatorname{sinc}\left(\frac{K_{z}}{2} z\right),
\end{aligned}
$$

where $\operatorname{sinc}(x)=\sin (x) / x$. The second derivative in the blurring function is introduced by the spreading loss factor $k_{z}^{2}$, however, its full width at half-maximum (FWHM) in the $z$ domain is close to that of the sinc function,

$$
z_{\mathrm{FWHM}} \approx \frac{0.6 c}{f L_{\sin \theta}},
$$

where $L_{\sin \theta}=K_{z} / k$ is the trace signal extent in $\sin \theta$. Figure 5 shows a simulated VTR in a noiseless environment for a source at $50 \mathrm{~m}$ depth that reaches CPA at $t_{\mathrm{CPA}}=60 \mathrm{~min}$. All other simulation parameters are listed in Table I. The effect of trace signal extent $L_{\sin \theta}$ on the depth estimate's resolution can be illustrated by limiting the extent of the trace signal supplied to the transform. This is shown in Fig. 6 for the cases of full, half, and quarter extents in $\sin \theta$ corresponding to the vertical double arrows in Fig. 5. The depth transform outputs in Fig. 6 illustrate the inverse relationship between $L_{\sin \theta}$ and $z_{\mathrm{FWHM}}$ indicated by Eq. (25).

Equation (25) allows setting geometrical constraints on target trajectories that determine the depth resolution in the transform output. For example, from the geometry in Fig. 1, $L_{\sin \theta}$ is given by 


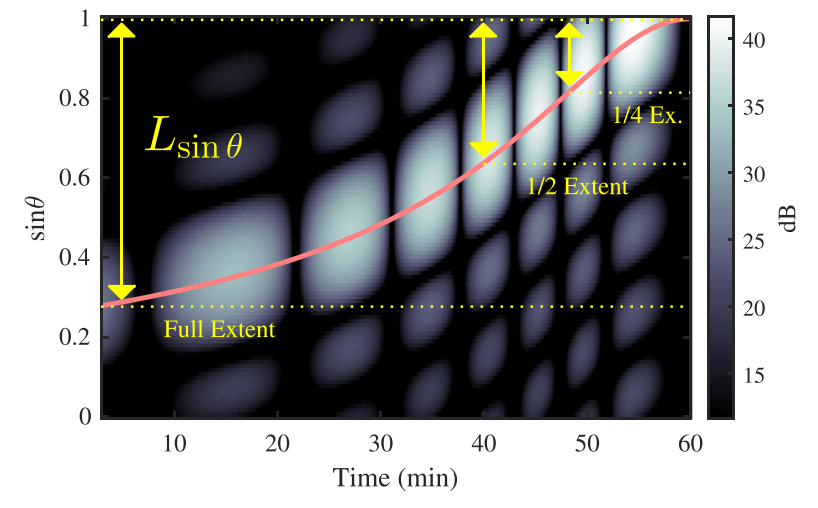

FIG. 5. (Color online) Simulated VTR for source at $50 \mathrm{~m}$ depth with overlays showing full extent $L_{\sin \theta}$ of the trace signal in $\sin \theta$ along with half and quarter extents. Trace signals corresponding to this VTR are used in the depth transform outputs in Fig. 6.

$$
L_{\sin \theta}=\frac{\bar{z}}{\sqrt{\bar{z}^{2}+r_{\mathrm{CPA}}^{2}}}-\frac{\bar{z}}{\sqrt{\bar{z}^{2}+r_{\max }^{2}}},
$$

where $r_{\max }$ is the maximum detectable range. Assuming $r_{\max }=15 \mathrm{~km}$, Eq. (26) can be substituted into Eq. (25), which allows computation of $z_{\mathrm{FWHM}}$ for a given frequency and $r_{\mathrm{CPA}}$, as shown in Fig. 7. The figure shows that a source emitting a $150 \mathrm{~Hz}$ signal detectable at $r_{\max }=15 \mathrm{~km}$ and passing within $5 \mathrm{~km}$ surface range of the VLA $\left(r_{\mathrm{CPA}}=5 \mathrm{~km}\right)$ would provide a depth resolution of $z_{\mathrm{FWHM}} \approx 15 \mathrm{~m}$. This assumes the depth-harmonic interference pattern is sufficiently sampled in the interval given by Eq. (26), the target trajectory requirements for which will be discussed in greater detail in Sec. IV A.

\section{B. Trace signal sampling and depth ambiguity}

The sampling of the trace signal in the $k_{z}$ domain described in Eq. (15) produces the second convolution term (20), which introduces ambiguity in the depth estimate in the form of aliasing in the $z$ domain. However, while the trace signal is uniformly sampled in time by the Dirac comb in Eq. (14), the relationship between $t$ and $\sin \theta_{s}(t)$ in Eq. (6) [and, therefore, $k_{z s}(t)$ ] is nonlinear. As a result of this nonlinearity, the sampling function (15) is a train of Dirac delta

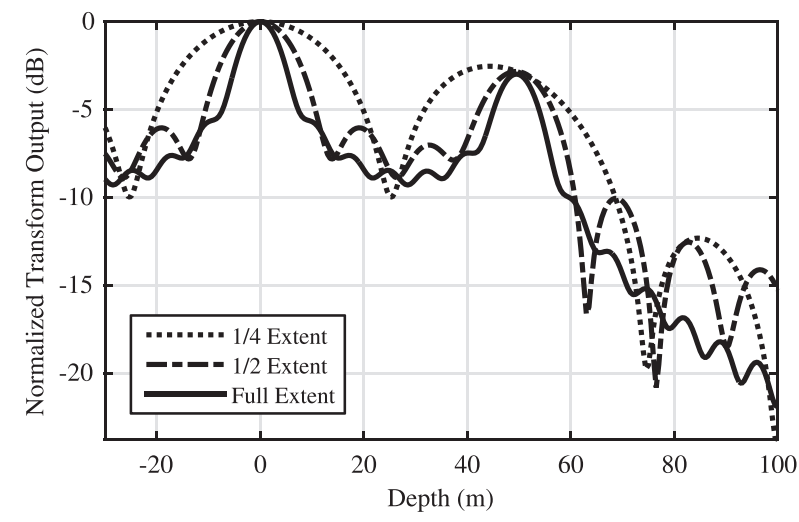

FIG. 6. Depth transform outputs for the three trace signal extents shown in Fig. 5 demonstrating the inverse relationship between trace signal extent $L_{\sin \theta}$ and depth resolution $z_{\mathrm{FWHM}}$ described in Eq. (25).

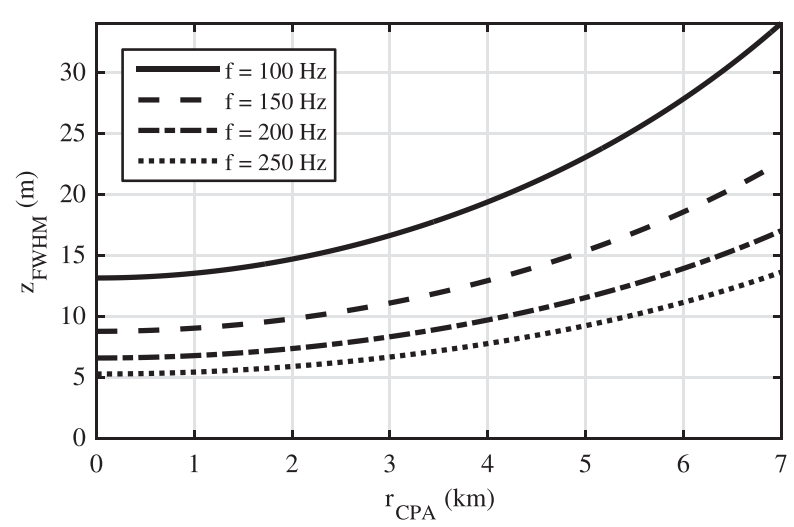

FIG. 7. Depth resolution $z_{\mathrm{FWHM}}$ as a function of surface range $r_{\mathrm{CPA}}$ assuming a maximum detectable range $r_{\max }$ of $15 \mathrm{~km}$ for a range of frequencies.

functions that are uniformly sampled in time, but nonuniformly sampled in the $k_{z}$ domain.

The sampling interval $\Delta t$ in Eq. (14) can be related to the sampling interval in $k_{z}$ using Eq. (11) and the time derivative of Eq. (6),

$$
\Delta k_{z} \approx \frac{\partial \sin \theta_{\hat{s}}(t)}{\partial t} k \Delta t=\frac{v_{s}^{2}\left(t_{\mathrm{CPA}}-t\right)}{\bar{z}^{2}} \sin ^{3} \theta_{\hat{s}}(t) k \Delta t .
$$

For a uniformly sampled function of $k_{z}$, the "Nyquist depth" would be inversely proportional to $2 \Delta k_{z}$. However, since the sampling in $k_{z}$ is nonuniform, the effective Nyquist depth is set by the largest sampling interval, ${ }^{15}$

$$
\begin{aligned}
\max \left[\Delta k_{z}\right] & \approx \max \left[\frac{\partial \sin \theta_{\hat{s}}(t)}{\partial t}\right] k \Delta t \\
& =\frac{2 \sqrt{3}}{9} \frac{\bar{z} v_{s}}{\bar{z}^{2}+r_{\mathrm{CPA}}^{2}} k \Delta t
\end{aligned}
$$

The Nyquist depth is then given by

$$
z_{\text {Nyquist }}=\frac{1}{2} \frac{2 \pi}{\max \left[\Delta k_{z}\right]}
$$

Figure 8 shows a plot of the Nyquist depth for a range of frequencies between 100 and $300 \mathrm{~Hz}$ and $r_{\mathrm{CPA}}$ ranging from 0 to $5 \mathrm{~km}$ assuming $c=1500 \mathrm{~m} / \mathrm{s}$ and the values of $\bar{z}$, $v_{s}$, and $\Delta t$ listed in Table I. Note the minimum effective Nyquist depth shown is $\sim 1.8 \mathrm{~km}$-well beyond the depth of interest for realistic targets.

\section{ESTIMATING DEPTH BY MEASURING NULL SPACING}

In this section, a new simplified method for estimating target depth is introduced. As mentioned in Sec. II, the presence of an interference structure in a trace signal indicates a target at depth. From Eq. (8), the nulls in the interference structure occur when the target is at vertical angles that are integral multiples of $\sin \theta_{\text {period }}$ in Eq. (9). Given a trace signal exhibiting this interference structure, the null period can be observed directly and used to estimate the source depth using 


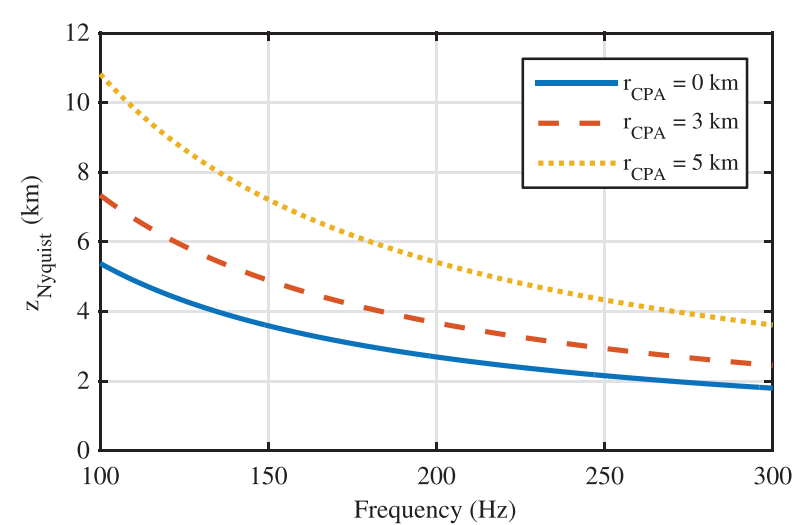

FIG. 8. (Color online) Effective Nyquist depth $z_{\text {Nyquist }}$ as a function of frequency calculated using Eq. (29) for a range of $r_{\mathrm{CPA}}$ values assuming $c=1500 \mathrm{~m} / \mathrm{s}$ and all other parameters listed in Table I.

$$
z_{s}=\frac{c}{2 f \sin \theta_{\text {period }}} .
$$

This simple method of measuring null spacing does not require the application and interpretation of the depth transform and its output. Its simplicity may be of advantage in a VTR cluttered by many surface interferers in which only a small number of nulls might be visible between the overlapping tracks.

In the case of an uncluttered target track, the target trajectory may limit the number of nulls observed in the trace signal. Section IV A describes how a target's trajectory is related to the trace signal observed in a VTR and establishes limits on trajectories that allow for observation of the depth-harmonic modulation. The ability to accurately measure the null period is then discussed in Sec. IV B as it relates to array aperture requirements for depth estimation.

\section{A. Target trajectory constraints}

From the geometry in Fig. 1, $\sin \theta$ at any surface range coordinate $r$ can be expressed as

$$
\sin \theta=\frac{\bar{z}}{\sqrt{r^{2}+\bar{z}^{2}}} .
$$

Substituting this expression for the target track, $\sin \theta_{s}(t)$, in Eq. (8) gives the TL as a function of a source's surface range,

$$
\operatorname{TL}(\omega, r)=-10 \log \left[\frac{B(\omega, \sin \theta)}{|S(\omega)|^{2}}\right] .
$$

Figure 9 shows a top-down view of the range-dependent TL from a target at $100 \mathrm{~m}$ depth and a frequency of $150 \mathrm{~Hz}$ assuming the array lies at a depth of $5 \mathrm{~km}$. Assuming a minimum of two periods of the harmonic structure (three adjacent nulls) need to be observed on the VLA for depth estimation, an upper limit on the range at CPA for a given source depth and frequency can be derived by first noting that the target's track will span vertical angles within the range

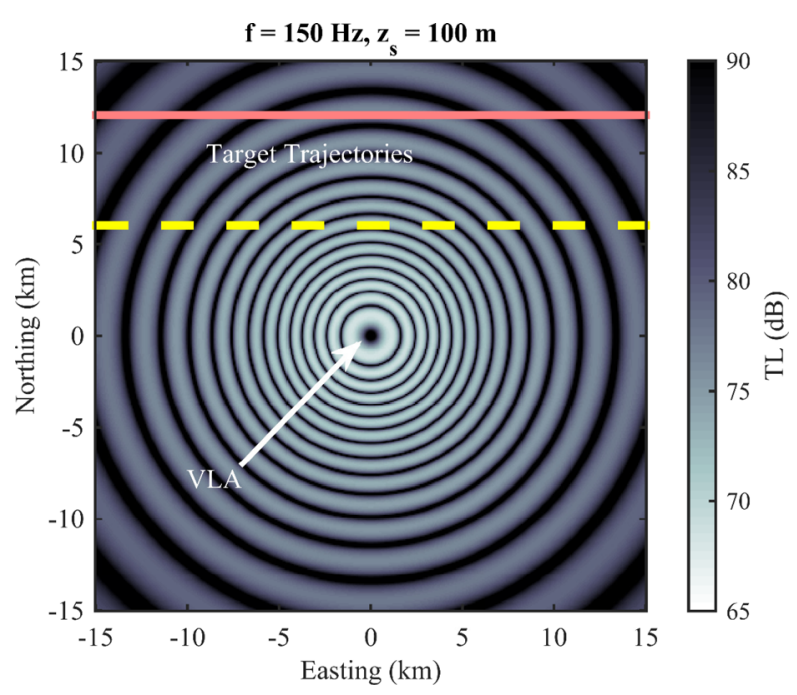

FIG. 9. (Color online) TL as a function of surface range. The VLA is positioned at the center of the figure. The horizontal lines crossing the plot represent two possible target trajectories with CPAs of $6.1 \mathrm{~km}$ (dashed) and $12.1 \mathrm{~km}$ (solid).

$$
\frac{\bar{z}}{\sqrt{r_{\max }^{2}+\bar{z}^{2}}} \leq \sin \theta \leq \frac{\bar{z}}{\sqrt{r_{\mathrm{CPA}}^{2}+\bar{z}^{2}}}
$$

with total span given by Eq. (26). Using Eqs. (9) and (33) and imposing the two period constraint, the upper limit on $r_{\mathrm{CPA}}$ for a given source depth and frequency can be expressed as

$$
r_{\mathrm{CPA}} \leq \bar{z} \sqrt{\left(\frac{c}{f z_{s}}+\frac{\bar{z}}{\sqrt{\bar{z}^{2}+r_{\max }^{2}}}\right)^{-2}-1 .}
$$

Figure 10 shows a contour plot of this upper limit as a function of frequency and $r_{\max }$ assuming an array whose center lies at depth $\bar{z}=5 \mathrm{~km}$. The maximum $r_{\mathrm{CPA}}$ in Eq. (34) depends on the product of the frequency and source depth.

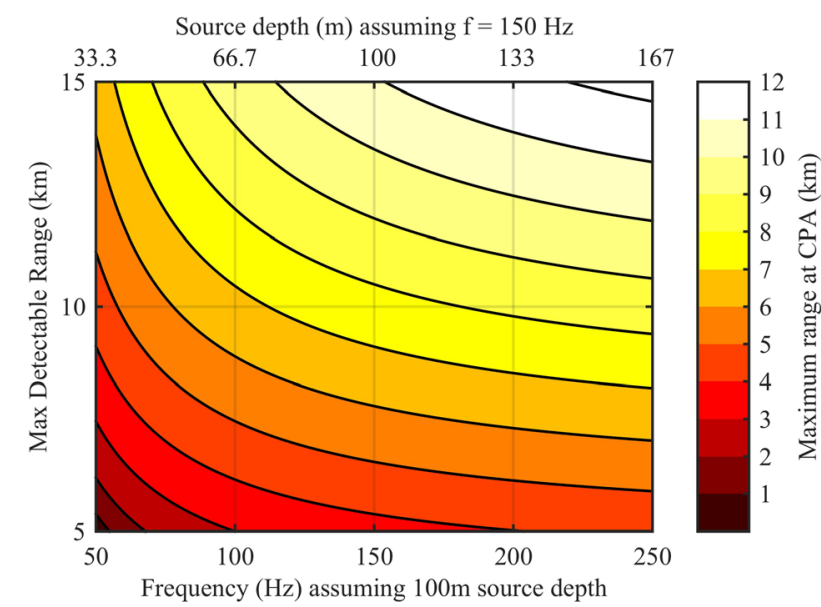

FIG. 10. (Color online) Plot of maximum CPA range, assuming two periods (three nulls) are required for depth estimation. From Eq. (34), the observed modulation is dependent on the product of source depth and frequency. Therefore, two different horizontal axes are provided, one with source frequency fixed at $150 \mathrm{~Hz}$ and varying source depth (upper axis), the other with depth fixed at $100 \mathrm{~m}$ and varying source frequency (lower axis). 
This allows the horizontal axis in Fig. 10 to be expressed either as a varying frequency and constant source depth (lower axis) or as a varying source depth at constant frequency (upper axis). From the lower axis of Fig. 10, it can be seen that a $150 \mathrm{~Hz}$ source at a depth of $100 \mathrm{~m}$ with sufficient source level to allow a maximum detectable range of $10 \mathrm{~km}$ would need to come within $\approx 7.6 \mathrm{~km}$ of the VLA for two periods of the depth-harmonic modulation to be observed.

\section{B. Aperture requirements}

The depth-harmonic interference pattern of a submerged source will be observed on any aperture, including a single hydrophone, provided the signal is received with sufficient signal-to-noise ratio (SNR) for detection. However, a single hydrophone element lacks the spatial sampling required to beamform the received signal and provide information about the directionality of the incident wavefronts in the $\sin \theta$ domain. As the depth-harmonic interference pattern is periodic in $\sin \theta$, a single hydrophone would therefore be unable to observe the periodicity in a signal from a submerged source. Furthermore, for a given inter-element spacing, longer arrays increase both the vertical aperture and the array gain (assuming uncorrelated noise). Both of these factors contribute to the accuracy of the resulting depth estimate. Conversely, shorter arrays with fewer elements provide less vertical aperture and array gain, which reduces the SNR and degrades the resolution in the vertical arrival angle estimate. Shorter arrays with fewer elements should therefore provide less accurate depth estimates. In the following analysis, the angular resolution is assumed to be the limiting factor that imposes a minimum number of hydrophones in the array. The accuracy in vertical angle estimation is therefore quantified using the $3 \mathrm{~dB}$ angular beam width ${ }^{16}$

$$
\theta_{3 \mathrm{~dB}}=\sin ^{-1}\left(\sin \theta+\frac{q \lambda}{2 N d}\right)-\sin ^{-1}\left(\sin \theta-\frac{q \lambda}{2 N d}\right)
$$

in this analysis, where $\lambda=c / f$ is the wavelength, $d$ is the array spacing, $N$ is the number of array elements, and the factor $q=0.89$ for uniform array shading.

Figure 11 shows a VTR generated using an image theory simulation with $c=1500 \mathrm{~m} / \mathrm{s}$ and all other parameters listed in Table I. The target trace is shown as a solid line with error bars representing $\pm \theta_{3 \mathrm{~dB}} / 2$, calculated using Eq. (35) at each interference null. Two horizontal dashed lines indicate adjacent interference nulls at $\sin \theta=0.9$ and 0.8 . The spacing between these nulls can be used to estimate the source depth using Eq. (30), however if the estimated location of one (or both) of the nulls is inaccurate due to a wide beam (poor angular resolution), the resulting estimate of the source depth will also be inaccurate. This can be illustrated by introducing error corresponding to $\pm \theta_{3 \mathrm{~dB}} / 2$ from Eq. (35) into the calculation in Eq. (30). For the example in Fig. 11, this becomes ${ }^{15}$

$$
z_{s}=\frac{c}{2 f\left[0.9-\sin \left(\sin ^{-1}(0.8) \pm \frac{\theta_{3 \mathrm{~dB}}}{2}\right)\right]},
$$

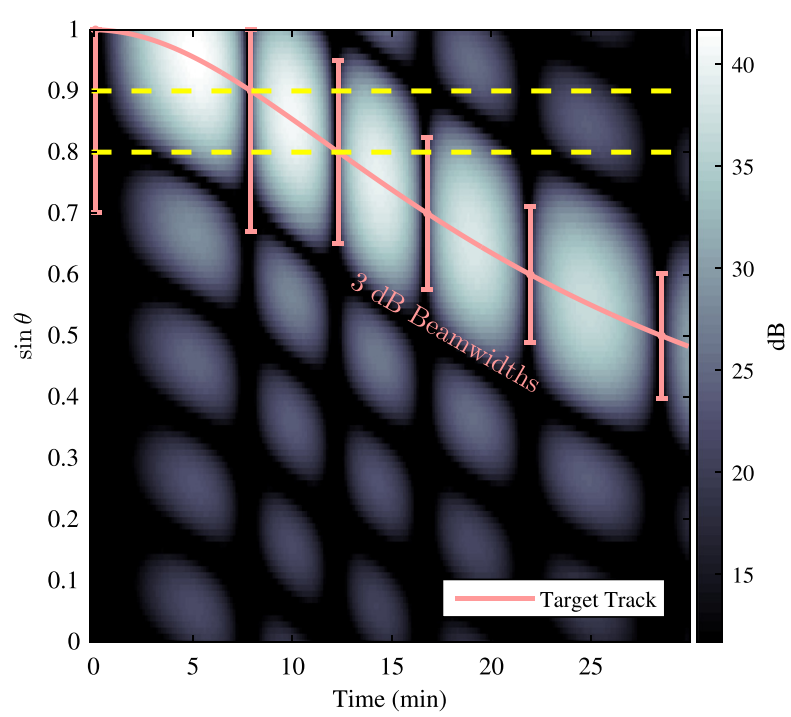

FIG. 11. (Color online) Illustration of using the $3 \mathrm{~dB}$ beam width to estimate the worst-case depth estimate. The simulated VTR was obtained from an image theory simulation for a $150 \mathrm{~Hz}$ source at $50 \mathrm{~m}$ depth received on a ten-element VLA. The dashed horizontal lines indicate the $\sin \theta$ locations of two adjacent nulls, while the true target track is shown as a solid line with error bars corresponding to $\pm \theta_{3 \mathrm{~dB}} / 2$ at each null.

where $\theta_{3 \mathrm{~dB}}$ corresponds to the $3 \mathrm{~dB}$ beam width centered at $\sin \theta=0.8$. The resulting depth estimates are plotted in Fig. 12 along with the ground truth. Figure 12 shows this method may produce a gross overestimate of source depth for a small number of hydrophones. However, from Fig. 11, the target track appears to be easily identifiable visually even with a wide beam, which suggests it may still be accurately estimated manually or by using an automated method. This tracking error based only on the array's $3 \mathrm{~dB}$ beam width is therefore likely to be a pessimistic worst-case estimate. Even so, while depth estimates with small arrays may lose accuracy, the presence of the depth-harmonic interference is still likely to be observable and indicate a submerged source without the need for a great degree of accuracy in estimated depth.

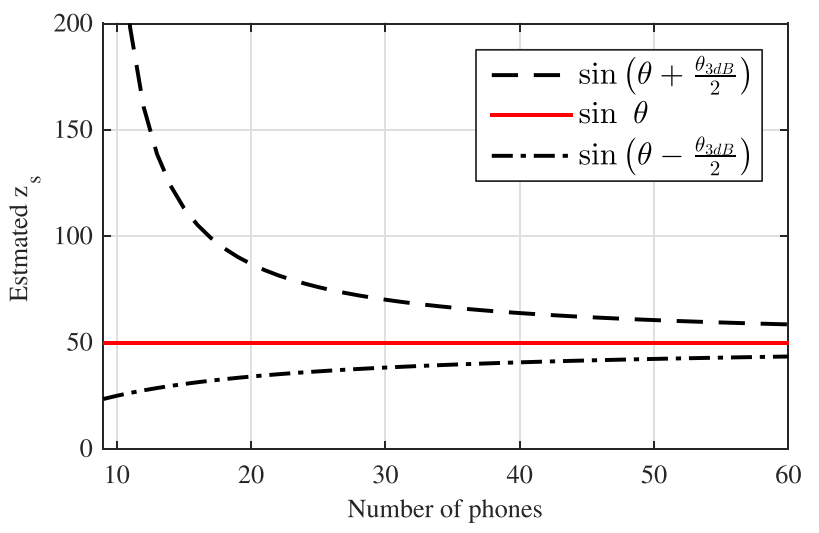

FIG. 12. (Color online) Error in depth estimate using Eq. (36), corresponding to the null at $\sin \theta=0.9$ in Fig. 11 and an erroneous value for the null at $\sin \theta=0.8$ as given by $\pm \theta_{3 \mathrm{~dB}}$, plotted as a function of number of phones $N$. Ground truth $\left(z_{s}=50 \mathrm{~m}\right)$ is given by the solid line, while error bounds corresponding to $\pm \theta_{3 \mathrm{~dB}} / 2$ in null location are given by the dashed lines. 


\section{SUMMARY}

This paper provides a detailed analysis of the factors impacting the performance of the recently introduced ${ }^{8}$ transform-based method for estimating the depth of submerged targets using VLAs positioned below the critical depth in the deep ocean. Such deep VLAs have shown the ability to utilize favorable propagation conditions that suppress noise from distant interferers and reduce TL from sources at moderate to close ranges. The transform-based method exploits the nearly ubiquitous depth-harmonic Lloyd's mirror interference pattern, which arises from the interaction of the direct and surface-reflected acoustic arrivals at low frequencies. The analysis presented in this paper shows the resolution in the transform-based depth estimate is inversely proportional to the extent of the target's track in vertical angle. Specifically, for a target at $50 \mathrm{~m}$ depth emitting a $150 \mathrm{~Hz}$ tone, $30 \mathrm{~m}$ resolution (FWHM) can be achieved with a target track encompassing as little as $11.5^{\circ}$ of angular extent. In addition, the ambiguity (aliasing) in the depth estimate, arising from the sampling of the signal over a finite set of vertical angles, is likely to have a negligible effect on the depth estimate for most manmade sources.

A simplified depth estimation method based on the angular spacing of the depth-harmonic interference nulls was also introduced and used as a basis for establishing target trajectory constraints for observation of the interference modulation, as well as array requirements for accurate depth estimation. The analysis presented shows that the modulation is likely to be observed for realistic target trajectories. Specifically, the modulation from a $150 \mathrm{~Hz}$ source at a depth of $100 \mathrm{~m}$ and with a maximum detectable range of $10 \mathrm{~km}$ would only need to come within $7.6 \mathrm{~km}$ of the VLA for the interference structure to be observable. In addition, VLAs with as few as ten elements (assuming sufficient SNR for detection) are likely to provide sufficient resolution to accurately distinguish surface interferers from submerged targets even if an accurate depth estimate cannot be obtained.

\section{ACKNOWLEDGMENTS}

This work was supported by the United States Office of Naval Research.

${ }^{1}$ Wenz, G. M., "Acoustic ambient noise in the ocean: Spectra and sources," J. Acoust. Soc. Am. 34(12), 1936-1956 (1962).

${ }^{2}$ Gaul, R. D., Knobles, D. P., Shooter, J. A., and Wittenborn, A. F., "Ambient noise analysis of deep-ocean measurements in the Northeast Pacific,” IEEE J. Oceanic Eng. 32(2), 497-512 (2007).

${ }^{3} \mathrm{Li}$, Z., Zurk, L. M., and Ma, B., "Vertical arrival structure of shipping noise in deep water channels," in OCEANS 2010 (IEEE, New York, 2010), pp. 1-8.

${ }^{4}$ Urick, R. J., Principles of Underwater Sound for Engineers (McGrawHill, New York, 1967), Chap. 6.

${ }^{5}$ McCargar, R. K. and Zurk, L. M., "Depth-based suppression of moving interference with vertical line arrays in the deep ocean," J. Acoust. Soc. Am. 132(3), 2081 (2012).

${ }^{6}$ Baggeroer, A. B., Kuperman, W. A., and Mikhalevsky, P. N., "An overview of matched field methods in ocean acoustics," IEEE J. Oceanic Eng. 18(4), 401-424 (1993).

${ }^{7}$ Zurk, L. M., Lee, N., and Ward, J., "Source motion mitigation for adaptive matched field processing," J. Acoust. Soc. Am. 113(5), 2719-2731 (2003). ${ }^{8}$ McCargar, R. and Zurk, L. M., "Depth-based signal separation with vertical line arrays in the deep ocean," J. Acoust. Soc. Am. 133(4), EL320-EL325 (2013).

${ }^{9}$ Zurk, L. M., Boyle, J. K., and Shibley, J., "Depth-based passive tracking of submerged sources in the deep ocean using a vertical line array," in 2013 Asilomar Conference on Signals, Systems and Computers (IEEE, New York), pp. 2130-2132 (2013).

${ }^{10}$ Reeder, D. B., "Clutter depth discrimination using the wavenumber spectrum," J. Acoust. Soc. Am. 135(1), EL1-EL7 (2014).

${ }^{11}$ Duan, R., Yang, K., Ma, Y., Yang, Q., and Li, H., "Moving source localization with a single hydrophone using multipath time delays in the deep ocean," J. Acoust. Soc. Am. 136(2), EL159-EL165 (2014).

${ }^{12}$ Jensen, F. B., Kuperman, W. A., Porter, M. B., and Schmidt, H., Computational Ocean Acoustics (Springer, New York, 2011), Chap. 5.

${ }^{13}$ Munk, W. H., "Sound channel in an exponentially stratified ocean, with application to SOFAR," J. Acoust. Soc. Am. 55(2), 220-226 (1974).

${ }^{14}$ Goodman, J. W., Introduction to Fourier Optics, 3rd ed. (Roberts and Company, Greenwood Village, CO 2004), Chap. 3.

${ }^{15}$ Boyle, J. K., "Performance metrics for depth-based signal separation using deep vertical line arrays," Master's thesis, Portland State University, Portland, OR, 2015.

${ }^{16}$ Van Trees, H. L., Detection, Estimation, and Modulation Theory, Optimum Array Processing (Wiley, New York, 2004), Chap. 2. 